\title{
Organization of Choline Acetyltransferase-Containing Structures in the Forebrain of the Rat
}

\author{
Tomoyuki Ichikawa and Yukio Hirata \\ Department of Anatomy and Embryology, Tokyo Metropolitan Institute for Neurosciences, Tokyo, Japan 183
}

The cholinergic structures in the forebrain of the rat were studied immunohistochemically with a monoclonal antibody to choline acetyltransferase (ChAT) which has proven to be particularly suited to reveal the trajectory of ChAT-positive fibers and their terminations.

The habenulo-interpeduncular tract contained the most distinctly ChAT-positive fibers. Here, ChAT-positivity was demonstrated in the perikarya, fibers and terminal field. From the basal forebrain, ChAT-positive fiber bundles could be followed (1) rostrally to the olfactory bulb, (2) dorsomedially to the neocortex and hippocampus, and (3) dorsocaudally to the hippocampus. Terminations of ChAT-positive fibers in these regions were either boutons terminaux (in the interpeduncular nucleus), finely granular (in the hippocampus) or a network of varicose fibers (in the olfactory bulb and neocortex).

Cholinergic terminal fields were also demonstrated in the following regions: the nucleus of the lateral olfactory tract, the olfactory tubercle, the pyriform cortex, the lateral nucleus and part of the basal nucleus of the amygdala, the caudate-putamen, the nucleus accumbens, some thalamic nuclei, and the suprachiasmatic nucleus of the hypothalamus. In most of these regions, ChAT-positivity appeared as either fine granules or a meshwork of varicose fibers.

The biosynthetic enzyme for ACh, choline acetyltransferase (ChAT), is currently the most reliable marker for cholinergic neurons (Fonnum, 1975; Kuhar, 1976; McCaman and McCaman, 1976). The distribution of the cholinergic perikarya in the mammalian CNS has been established immunohistochemically using antisera (Kimura et al., 1981a, b; Sofroniew et al., 1982) or monoclonal antibodies (Armstrong et al., 1983; Hedreen et al., 1983; Houser et al., 1983; Mesulam et al., 1984; Nagai et al., 1983; Satoh et al., 1983) to ChAT. ChAT immunohistochemistry has revealed that the histochemical demonstration of AChE, which has been widely used for studying cholinergic neurons, can be adopted only in some regions in the CNS (Eckenstein and Sofroniew, 1983; Levey et al., 1983; Mesulam et al., 1984; Satoh et al., 1983). Substantial evidence concerning the ascending cholinergic pathways in the CNS has also been derived from the immunohistochemical studies of ChAT in combination with tracer transport (Mesulam et al., 1983a, b; Nagai et al., 1982; Saper, 1984; Wahle et al., 1984; Wainer and Rye, 1984; Woolf et al., 1983). Using such a technique, Mesulam et al. (1983b) argued cogently that the rat ol-

\footnotetext{
Received Dec. 10, 1984; revised July 25, 1985; accepted July 31, 1985.

We wish to thank Mrs. Kyoko Ajiki for her excellent technical assistance. This work was supported in part by Grants-in-Aid for Scientific Research from the Ministry of Education, Science and Culture of Japan.

Correspondence should be addressed to Tomoyuki Ichikawa, Department of Anatomy and Embryology, Tokyo Metropolitan Institute for Neurosciences, 2-6 Musashidai, Fuchu City, Tokyo, Japan 183.

Copyright (c) 1986 Society for Neuroscience $0270-6474 / 86 / 010281-12 \$ 02.00 / 0$
}

factory bulb, amygdaloid complex, hippocampal formation, neocortex and thalamic nuclei receive cholinergic innervation from six major regions of ChAT-positive neurons in the basal forebrain and upper brain stem. It is a prerequisite for understanding the organization of the central cholinergic system to trace ChAT-positive fiber pathways in addition to demonstrating ChAT positive perikarya and to determine the organization of ChAT-positive terminal fields. So far, however, there is no report on ChAT-positive fiber systems and only limited data on ChAT-positive structures in the neuropil (Chao et al., 1982; Houser et al., 1983; Kimura et al., 1981a, 1983).

We recently established monoclonal antibodies to rat ChAT (Ichikawa et al., 1983) and demonstrated the specificity of one of them (Ishida et al., 1983). Using it, we immunohistochemically investigated ChAT-positive fibers and the appearance of ChAT-positive structures in the neuropil of the rat forebrain.

\section{Materials and Methods}

\section{Monoclonal antibody}

Details of the production and characterization of monoclonal antibodies to ChAT have been described previously (Ichikawa et al., 1983). Briefly, a BAI.B/c mouse was immunized with partially purified ChAT from rat brain, and spleen cells of the mouse were fused with NS-1 myeloma cells. The culture medium of the hybrids was mixed with rat ChAT preparation and tested for the ability to remove ChAT activity from the supernatant after an indirect immunoprecipitation. Two relevant cell lines secreting antibodies, $A b-57$ and $A b-60$, respectively, were oblained. Both antibodies were IgG1 subclass immunoglobulins and showed a cross-species reactivity with $\mathrm{ChAT}$, from mouse and cat. Affinity of Ab-57 was, however, 100 times higher than that of Ab-60. The specificity of the former was confirmed by the immunoblotting technique after polyacrylamide gel electrophoresis, combined with a direct assay of ChAT activity in the gel; and by immunohistochemistry, which revealed ChAT-positive reactions in motoneurons known to be cholinergic (such as those in the brainstem and spinal cord) (Ishida et al., 1983). Ah-57, purified from ascitic fluid by a protein A-Sepharose column, was used in the present study.

\section{Tissue preparation}

Twenty Sprague-Dawley rats $(200-300 \mathrm{gm})$ were anesthetized with sodium pentobarbital $(50 \mathrm{mg} / \mathrm{kg})$ and perfused through the aortic cone with $0.1 \mathrm{M}$ phosphate buffer (PB), pH 7.4, containing $0.9 \% \mathrm{NaCl}$ (PBS) at room temperature, followed by $4 \%$ paraformaldehyde and $7.5 \%$ sucrose in $0.05 \mathrm{M} \mathrm{PB}$ with or without $0.1 \%$ glutaraldehyde, at $4^{\circ} \mathrm{C}$ and at a flow rate of $15 \mathrm{ml} / \mathrm{min}$ for $15-20 \mathrm{~min}$. Brains were immediately removed and the forebrain regions were cut serially on a freezing microtome at $40 \mu \mathrm{m} ; 14$ brains were sectioned in the frontal plane and six in the sagittal plane. Sections were collected in PBS, and every 5th frontal or sagittal section was processed for immunohistochemical labeling; a series of adjacent sections were stained with cresyl violet. Some additional sections served as controls in which Ab-57 was omitted or substituted by normal mouse IgG.

The fixative was a modification of the one previously used (Ishida et al., 1983), which consisted of $4 \%$ paraformaldehyde and $0.1 \%$ glutaraldehyde in $0.1 \mathrm{M}$ PB. Addition of sucrose to the fixative resulted in 
Figure 1. ChAT-positive structures in the olfactory brain. $A$, ChAT-positive varicose fibers are observed in the glomerular $(G l)$, external plexiform $(P l)$, mitral cell $(M i)$ and granule cell $(G r)$ layers of the olfactory bulb. The olfactory nerve layer $(O N)$ is free of staining $(\times 100)$. $B$, Pericellular distribution of ChAT-positive varicose fibers in the glomerular layer $(\times 400)$. $C$, The fibrillar layer of the anterior olfactory nucleus (arrows) is ChATpositive, while the lateral olfactory tract $(L O T)$ is free of staining. $C P$, caudate-putamen; $O T$, olfactory tubercle $(\times 10) . D$, Interwoven ChATpositive varicose fibers make the nucleus of the lateral olfactory tract (NLOT) distinct from the surroundings. $C h$, optic chiasm; $M P$, magnocellular preoptic nucleus $(\times 15) . E$, Fine granular ChAT-positivity in the molecular $(\mathrm{Mo})$ and polymorphic cell $(P o)$ layers of the olfactory tubercle. Strongly ChAT-positive perikarya are present mostly in the polymorphic cell layer. $P y$, pyramidal cell layer $(\times 75)$. $F$, Pericellular distribution of ChATpositive varicose fibers in the polymorphic cell $(P o)$ and pyramidal cell $(P y)$ layers in the pyriform cortex. A moderately ChAT-positive neuron is seen in the molecular layer $(\mathrm{Mo})$ $(\times 300)$.

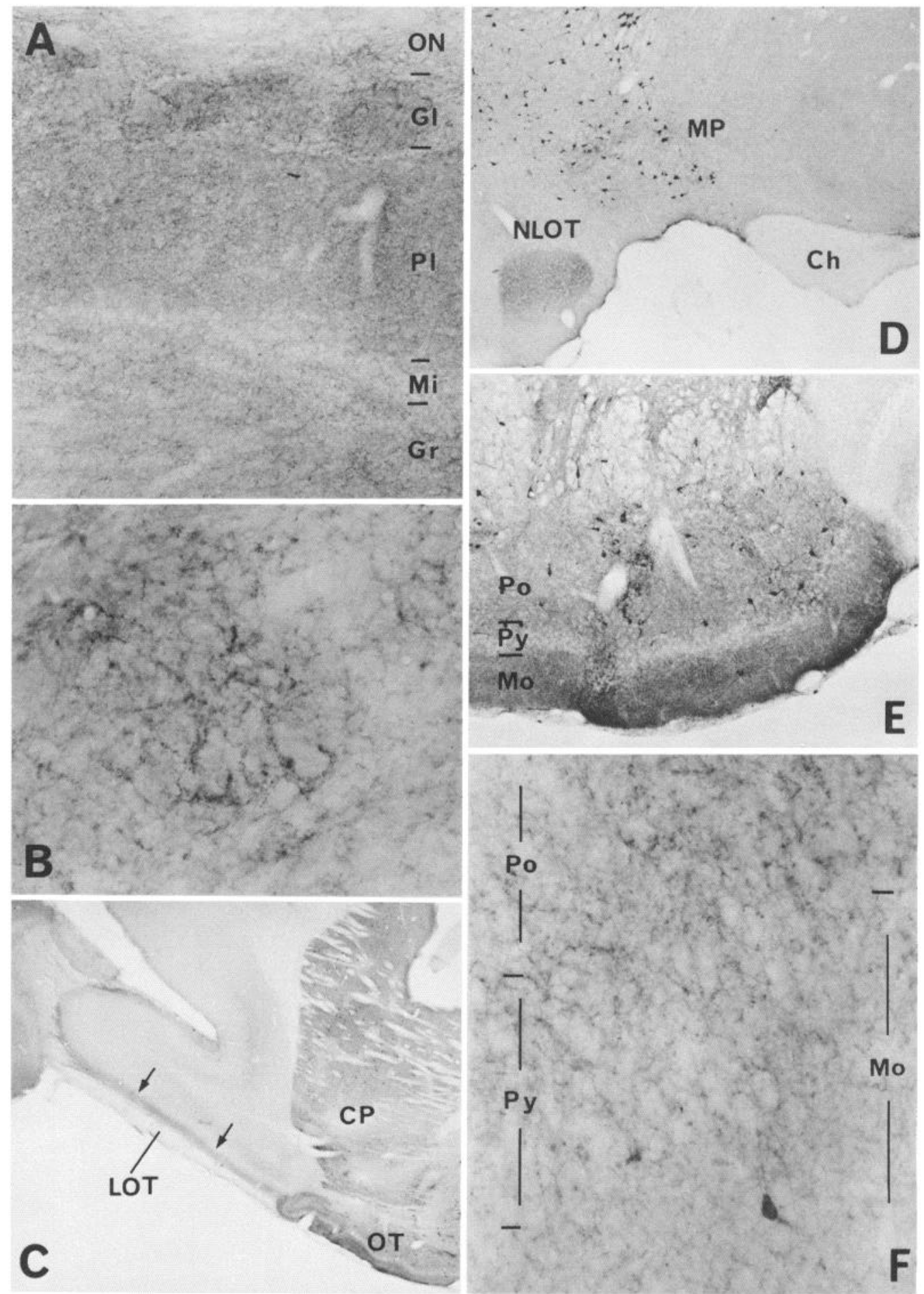

substantial prevention of damage during the freezing procedure. Glutaraldehyde tended to decrease the staining of ChAT-positive structures in the neuropil in several regions, but it also suppressed the staining of the background. Therefore, both fixatives with and without glutaraldehyde were used in the present study.

\section{Immunohistochemistry}

The avidin-biotin-peroxidase complex (ABC) method (Hsu et al., 1981) using Vectastain (Vector Laboratories) was adopted. The staining procedure consisted of the following incubation steps: (1) either Ab-57 or normal mouse IgG $(2.5-5 \mu \mathrm{g} / \mathrm{ml})$ in PBS containing $0.1 \%$ Triton X-100 (PBS-T), or PBS-T alone for $1 \mathrm{hr}$ at room temperature and then for an additional $16-18 \mathrm{hr}$ at $4^{\circ} \mathrm{C}$; (2) biotinylated horse anti-mouse IgG diluted 1:200 with PBS-T for $1 \mathrm{hr}$; (3) complex of avidin and biotinylated HRP diluted 1:100 with PBS-T for $30 \mathrm{~min}$; and (4) $0.05 \%$ 3,3'-diaminobenzidine tetrahydrochloride and $0.01 \% \mathrm{H}_{2} \mathrm{O}_{2}$ in $0.1 \mathrm{M} \mathrm{PB}$ for $10 \mathrm{~min}$. Sections were free-floating, and incubations carried out at room temperature unless otherwise specified. The sections were rinsed for $30 \mathrm{~min}$ with PBS-T between each step. After the final rinse with $0.1 \mathrm{M} P B$, sections were mounted on slides in gelatin-alcohol solution, and processed by standard procedures for light-microscopic observations.

The unlabeled peroxidase-antiperoxidase (PAP) method used previously (Ishida et al., 1983) was replaced by the ABC method in the present study. The latter method, in combination with the modified fixative, permitted more distinct visualization of ChAT-positive fibers 


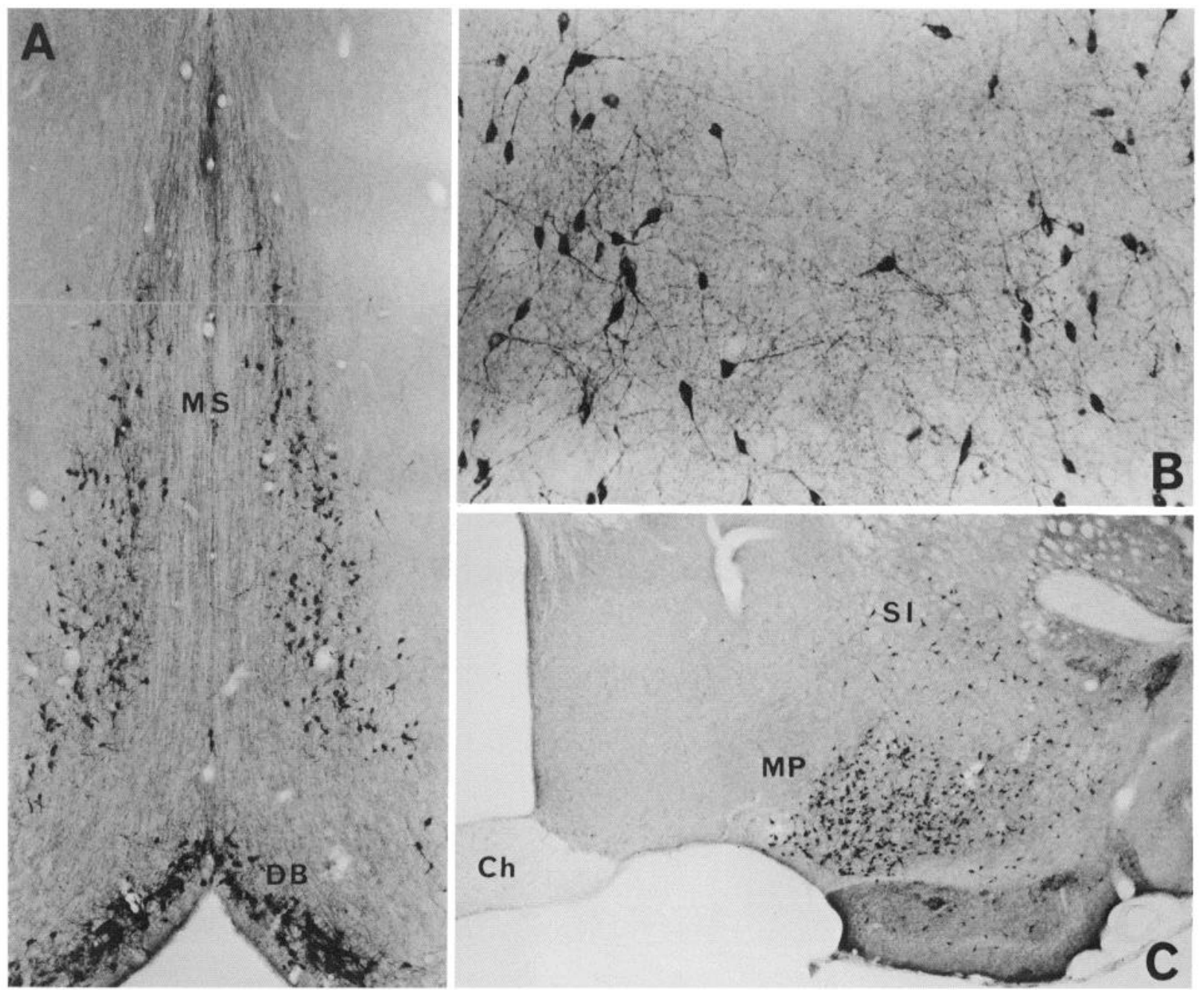

Figure 2. ChAT-positive structures in the basal forebrain. $A$, Many perikarya and axons in the nucleus of the diagonal band $(D B)$ and medial septal nucleus $(M S)$ are strongly ChAT-positive $(\times 30) . B$, ChAT-positive perikarya in the medial septal nucleus in higher magnification. These cells give off fine beaded processes $(\times 300)$. $C$, ChAT-positive perikarya in the magnocellular preoptic nucleus $(M P)$ and substantia innominata $(S I)$. Ch, optic chiasm $(\times 15)$.

and terminal-like structures with lower concentrations of the antibody than is obtainable with the PAP method.

\section{Results}

\section{Olfactory brain}

In the olfactory bulb, no ChAT-positive perikarya were observed. ChAT-positive varicose fibers were observed in the glomerular, external plexiform, mitral cell, and granule cell layers (Fig. 1 $A$ ). The olfactory nerve and medullary layers were free of staining. The varicose fibers in the glomerular layer showed a pericellular distribution (Fig. $1 B$ ), while those in other layers were scattered diffusely in the neuropil without any preferential association with specific elements. The lateral olfactory tract was free of staining over its full length, but the fibrillar layer of the anterior olfactory nucleus contained ChAT-positive, tangentially running fibers (Fig. $1 C$ ). These are a part of the rostral system, which connects ChAT-positive perikarya in the olfactory tubercle and basal forebrain with the ChAT-positive terminal field of the olfactory bulb.

Interwoven varicose fibers with ChAT-positivity made the nucleus of the lateral olfactory tract distinct from its surroundings (Fig. 1D), although the origin of these fibers was unknown. The varicosities in the nucleus were frequently in contact with perikarya. The molecular and polymorphic cell layers of the olfactory tubercle showed an intense ChAT-positivity which appeared mostly as fine granular profiles (Fig. $1 E$ ), but their structural details and origins could not be resolved. A small number of strongly ChAT-positive perikarya were present, mostly in the polymorphic cell layer (Fig. $1 E$ ). As for the pyriform cortex, ChAT-positive varicose fibers were observed in all layers, and the varicosities showed a pericellular distribution (Fig. $1 F$ ). A few ChAT-positive stellate cells were present in the molecular (Fig. $1 F$ ) and polymorphic cell layers.

\section{Basal forebrain}

The neuropil of this region was free from ChAT-positivity except for fibrous structures seemingly arising from strongly ChATpositive neuronal perikarya that were present in the nucleus of the diagonal band of Broca (Fig. $2 A$ ), the medial septal nucleus (Fig. 2, $A$ and $B$ ), the substantia innominata (Fig. 2C) and the magnocellular preoptic nucleus (Fig. $2 C$ ). Numerous vertically oriented ChAT-positive axons were observed in the vertical limb of the nucleus of the diagonal band and medial septal nucleus (Fig. 2A). ChAT-positive fine processes of the neurons in the basal forebrain were more or less beaded; this feature was most prominent in neurons of the medial septal nucleus (Fig. $2 B)$. 

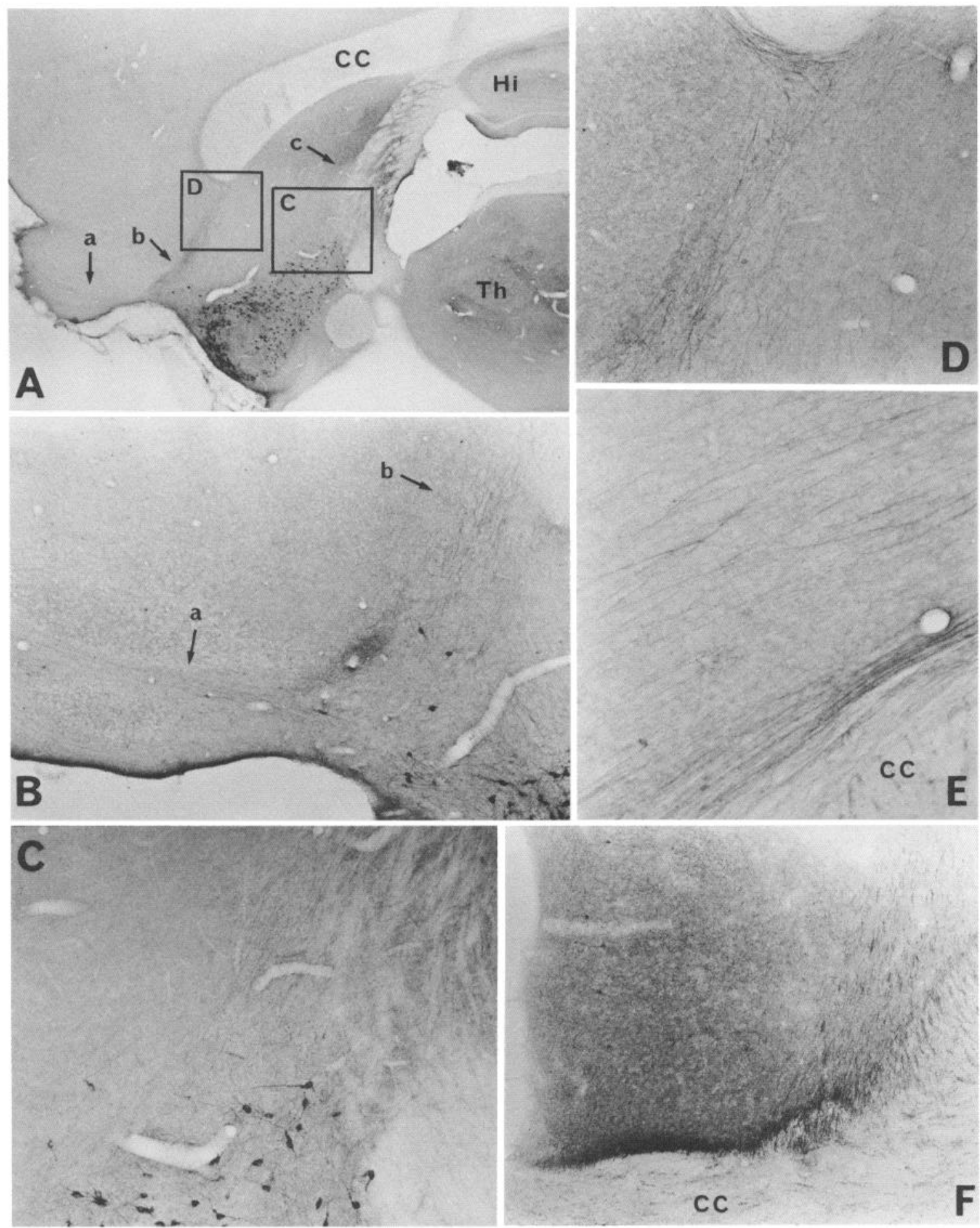

Figure 3. ChAT-positive fiber bundles from the basal forebrain to the olfactory bulb, neocortex and hippocampus. A, Low-power micrograph of a sagittal section showing the rostral system, $a$, to the olfactory bulb; the dorsomedial system, $b$, to the neocortex and hippocampus; and dorsocaudal system, $c$, to the hippocampus. Boxed areas $(C$ and $D)$ are reproduced at higher magnification. $C C$, corpus callosum; $H i$, hippocampus; $T h$, thalamus $(\times 15)$. $B$, ChAT-positive fiber bundle from the basal forebrain bifurcates at the rostral aspect of the nucleus of the diagonal band to give rise to the rostral system, $a$, through the medial olfactory tract; and the dorsomedial system, $b$, toward the genu of the corpus callosum ( $\times 50$ ). $C$, ChAT-positive fibers in the dorsocaudal system $(\times 75), D, E, F$, ChAT-positive fibers in the dorsomedial system. The ChAT-positive bundle divides into two groups at the genu of the corpus callosum $(D)(\times 75)$. One group runs over the corpus callosum $(C C)$ and some fibers enter the cingulate cortex directly $(E)(\times 150)$. ChAT-positive fibers entering the cingulate cortex and adjoining dorsal neocortex $(F)($ frontal section, $\times 75)$. Some ChAT-positive perikarya are observed in the cingulate cortex. CC, corpus callosum. 
Three ChAT-positive fiber systems arising from the basal forebrain were identified:(1) A rostral system consisting of ChATpositive fibers running toward the olfactory bulb through the fibrillar layer of the anterior olfactory nucleus (Fig. 1C) and through the medial olfactory tract (Fig. 3, $A$ and $B$ ). (2) A dorsomedial system in which fibers proceed dorsally from the rostral aspect of the nucleus of the diagonal band up to the genu of the corpus callosum, where they bifurcate. Many fibers turn caudally over the genu of the corpus callosum but some run caudally beneath it (Fig. 3, $A$ and $D$ ). Most fibers taking the dorsal route run caudally into the cingulate bundle. Many of these fibers leave the bundle and enter the cingulate cortex (Fig. $3, E$ and $F$ ) and the adjoining dorsal neocortex, but some can be traced further through the bundle to the splenium of the corpus callosum, where they turn ventrally and seem to sweep into the hippocampal formation. Fibers taking the course beneath the corpus callosum run caudally through the dorsal fornix into the hippocampus together with the fibers in the dorsocaudal system, to be described below. (3) A dorsocaudal system, where ChAT-positive fibers run up dorsocaudally through the medial septal nucleus and dorsal fornix, and enter the hippocampus (Fig. 3, $A, C$ ).

\section{Amygdaloid complex}

ChAT-positive fibers emerging from the external capsule entered the posterior part of the lateral nucleus and the lateral large-celled part of the basal nucleus (Fig. 4). In the former nucleus, ChAT-positive varicose fibers were observed (Fig. $4 A$ ), while in the latter, ChAT-positivity was granular (Fig. $4 B$ ). In both nuclei, ChAT-positivity did not show any preferential association with specific neuronal elements. All other nuclei were free of staining (see Fig. $7 \mathrm{C}$ )

\section{Hippocampal formation}

A considerable number of strongly ChAT-positive perikarya were found in the superficial half of the cortical width of the presubiculum (Fig. 5, $A, D$ ). The ChAT-positivity of these, however, could not be traced either through dendrites or axons beyond the superficial half of the cortical depth. The neuropil of the area inhabited by ChAT-positive neuronal perikarya showed markedly reduced ChAT-positivity as compared with other cortical areas (Fig. 5, $A, D$ ).

In the subiculum, many ChAT-positive fibers were observed traversing the subcortical white matter through the subicular cortex, first obliquely and then tangentially, and many of these fibers could be followed toward the hippocampal fissure, where some fibers took a further tangential course into the superficial part of the molecular layer of Ammon's horn while others crossed the fissure to enter the dentate fascia (Fig. 5E).

In the hippocampal formation proper, finely granular ChATpositivity showed a characteristic areal and zonal distribution (Fig. 5, $A, B$ ), in contrast to the subicular area, where the positivity was present throughout the cortical thickness (Fig. $5 A$ ). In Ammon's horn, positivity was seen preferentially in the stratum oriens and the most superficial portion of the molecular layer, i.e., the stratum lacunosum moleculare, where ChATpositive fibers were seen in addition to granular positivity (Fig. $5 B)$. ChAT-positive grains in the stratum oriens were denser over the intermediate sector of Ammon's horn (CA2 to CA3) than others. The granular positivity of the stratum oriens continued to ChAT-positive fibers in the alveus and fimbria (Fig. $5 C$ ). These fibers are considered to belong to the dorsomedial and dorsocaudal systems from the basal forebrain. In the dentate fascia, ChAT-positive grains and varicosities surrounded perikarya of granule cells of both dorsal and ventral leaves, but the finely granular positivity in the molecular layer was restricted to its superficial two-thirds (outer molecular layer) of the dorsal leaf of the dentate fascia (Fig. 5B).

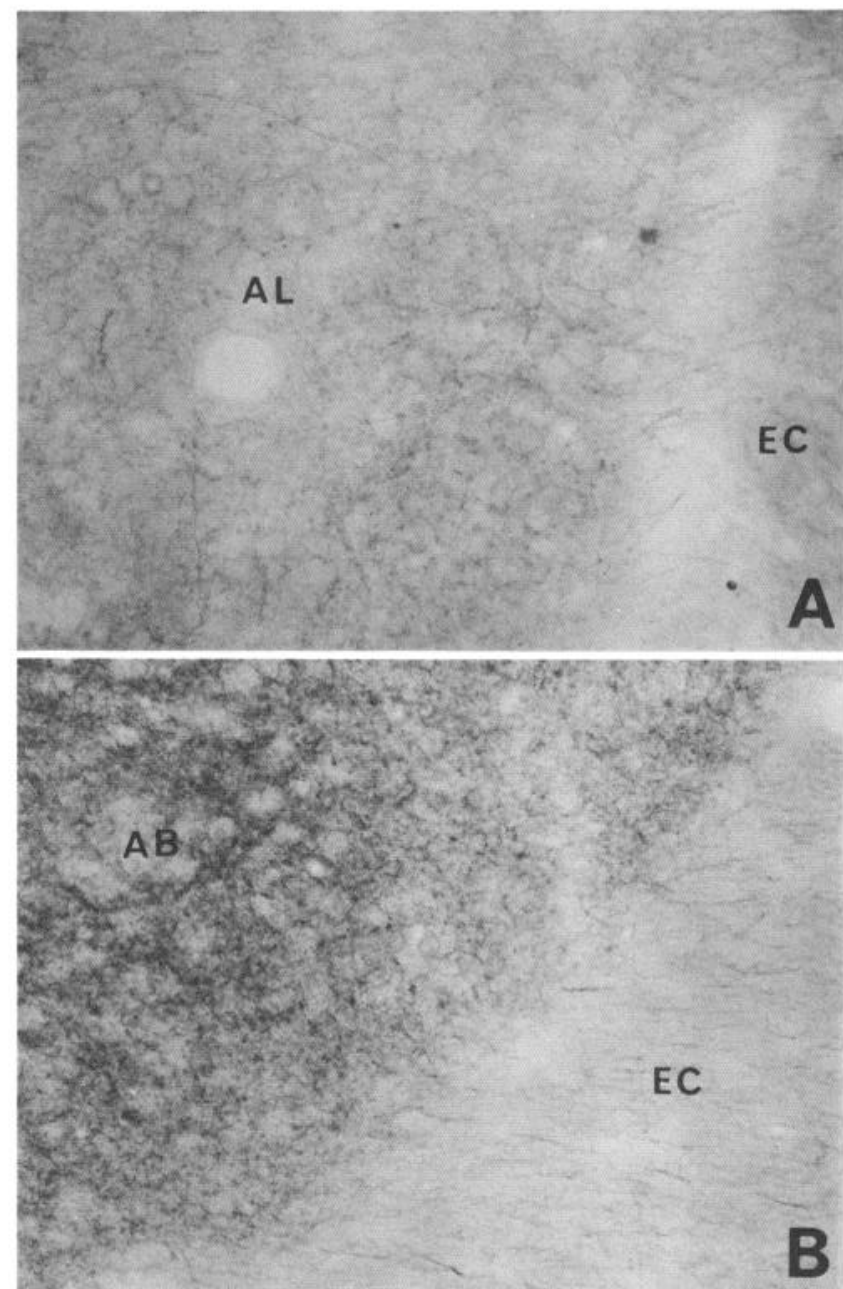

Figure 4. ChAT-positive structures in the amygdala. $A$, Varicose fibers in the posterior part of the lateral nucleus $(A L)$ are ChAT-positive. The fibers are continuous to ChAT-positive fibers in the external capsule $(E C)(\times 200), B$, ChAT-positive fibers in the external capsule $(E C)$ continue to ChAT-positive granular profiles in the lateral, large-celled part of the basal nucleus $(A B)(\times 200)$.

\section{Neocortex}

A ChAT-positive varicose fiber meshwork was present in the neocortex (Fig. 6B). The meshwork was continuous throughout all cortical layers without a conspicuous interlayer or interarea difference. The varicosities were scattered diffusely through the neuropil and were not preferentially associated with the cortical neuronal perikarya. Though some of the varicosities in the cingulate cortex and the adjoining neocortex were found to be derived from the cingulate bundle (Fig. $3, E, F$ ), the source of most of the neocortical varicosities could not be identified.

ChAT-positive neurons were present in layers II-VI, particularly in layers II and III (Fig. 6A). They represented only a small fraction of cortical neurons and the staining of these was markedly weaker than that of neurons in the basal forebrain. Many of the neurons with ChAT-positivity exhibited a bipolar dendritic pattern, while some were stellate type (Fig. $6 A$ ). Vertically oriented dendrites extended across several cortical layers.

\section{Caudate-putamen and nucleus accumbens}

ChAT-positive fine granular structures similar to those seen in the olfactory tubercle and lateral part of the basal amygdaloid nucleus were observed in the neuropil (Fig. $7 A$ ) throughout these two nuclei without a definitive boundary. ChAT-positive peri- 
Figure 5. ChAT-positive structures in the hippocampal formation. $A, \mathrm{~A}$ low-power micrograph of a sagittal section showing the characteristic areal and zonal distribution of ChAT-positivity in the hippocampal formation and presubiculum. The presubicular area contains ChAT-positive perikarya (arrows) $(\times 30)$. ChAT-positive fibers (arrowheads) entering the stratum oriens from the fornix $(F x)$ are shown in higher magnification in $C$ ( $\times 60)$. Boxed area is shown in E. B, Characteristic zonal distribution of ChAT-positivity in the hippocampal formation. $G r$, stratum granulosum; $L a$, stratum lacunosum-moleculare; $M E$, stratum moleculare externum; $M I$, stratum moleculare internum; $O r$, stratum oriens; $P y$, stratum pyramidale; $R a$, stratum radiatum $(\times 150)$. $D$, ChAT-positive perikarya in the presubiculum. The neuropil of this area shows markedly reduced ChATpositivity $(\times 110)$. $E$, Part of ChATpositive fibers tangentially running in the subiculum cross the hippocampal fissure $(H F)$ and enter the dentate fascia $(D t)(\times 90)$
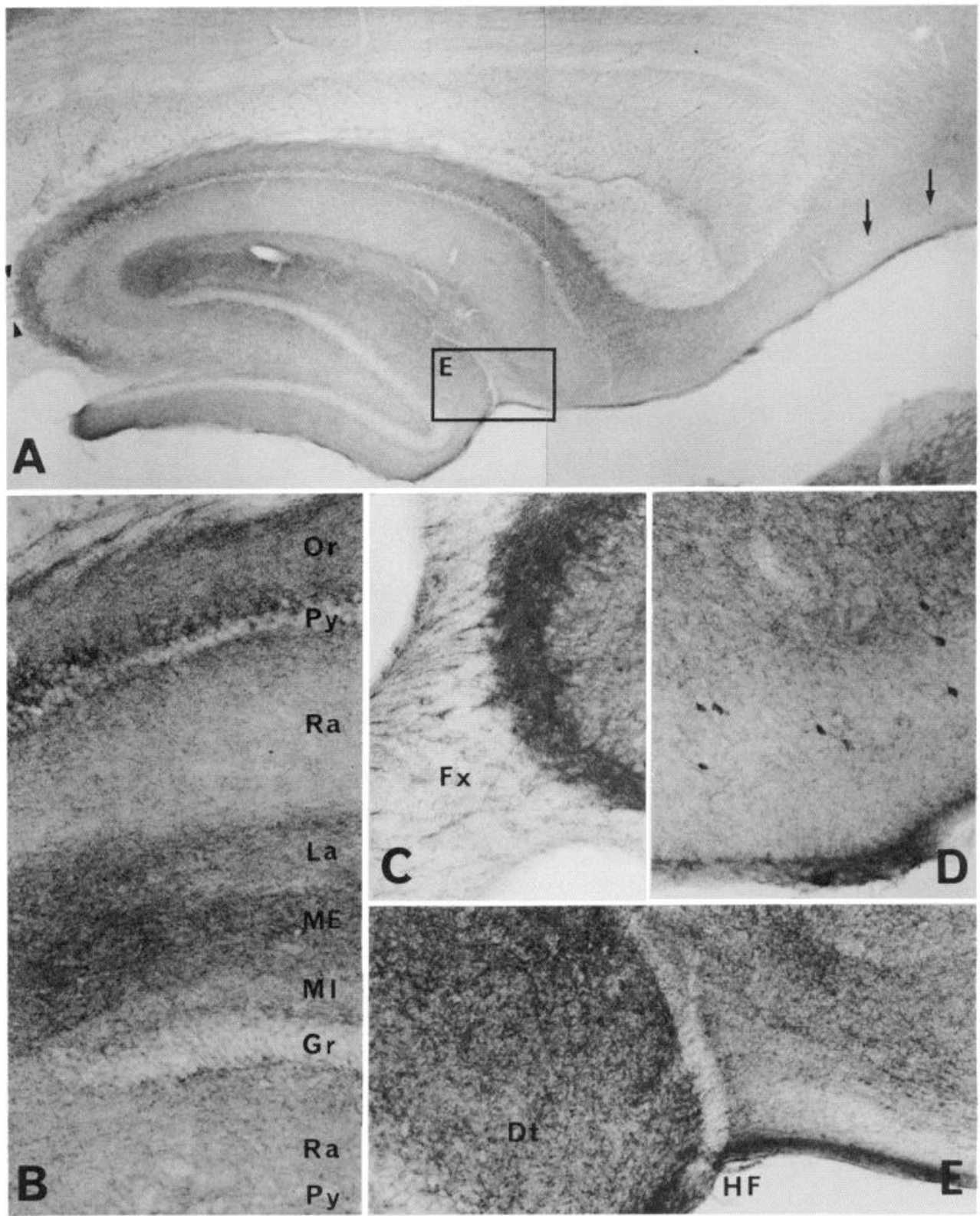

karya, oval or fusiform in shape, were scattered diffusely through the nuclei (Fig. 7B). Labeled neurons (10-15 $\times 20-35 \mu \mathrm{m})$ in the caudate-putamen represented a small portion of the total number of neurons. ChAT-positive fibers traversing the external capsule were followed to the putamen (Fig. $7 A$ ), although the direction of the fibers was not determined.

\section{Globus pallidus}

There were no ChAT-positive profiles in the neuropil except for dendrites and axons of strongly ChAT-positive perikarya (Fig. 7C). ChAT-positive fibers arising from the nucleus could be traced through the ventral surface of the cerebral peduncle toward the ventrolateral nucleus of the thalamus (Fig. 7, $C, D$ ), but the exact destination could not be ascertained. A few elongated ChAT-positive perikarya resembling ChAT-positive ones of the globus pallidus were present among the fiber bundle (Fig. 7D).

\section{Habenular and interpeduncular nucleus}

While there was no ChAT-positivity in the lateral habenular nucleus, moderately ChAT-positive perikarya were present in the medial habenular nucleus (Fig. $8 A$ ), but the neuropil was free of staining. A strongly ChAT-positive fiber bundle emerging from the ventrolateral aspect of the medial habenular nucleus descended ventrocaudally and entered the interpeduncular nucleus (Fig. $8 C$ ) through the fasciculus retroflexus (Fig. $8 \mathrm{~B}$ ). After entering the nucleus, ChAT-positive fiber bundles traversed the nucleus repeatedly, and numerous ChAT-positive terminal-like dots were observed budding from the fibers (Fig. $8 D$ ).

\section{Thalamus}

No ChAT-positive neurons were found in the dorsal thalamic nuclei. ChAT-positivity in this brain region was either in the form of straight to varicose fibrous profiles or of fine grains in the neuropil of some thalamic nuclei. The most marked ChATpositivity was in the thalamic reticular nucleus and in the anteroventral nucleus (Fig. 9, $A-C$ ), while the paracentral and reuniens nuclei and the dorsal anterior pretectal area were moderately ChAT-positive. ChAT-reaction of the reticular nucleus was more intense in the rostral half of its rostro-caudal extent. Finely granular positivity of this nucleus seemed to continue ventrally into the zona incerta. Some ChAT-reactive fibers ap- 


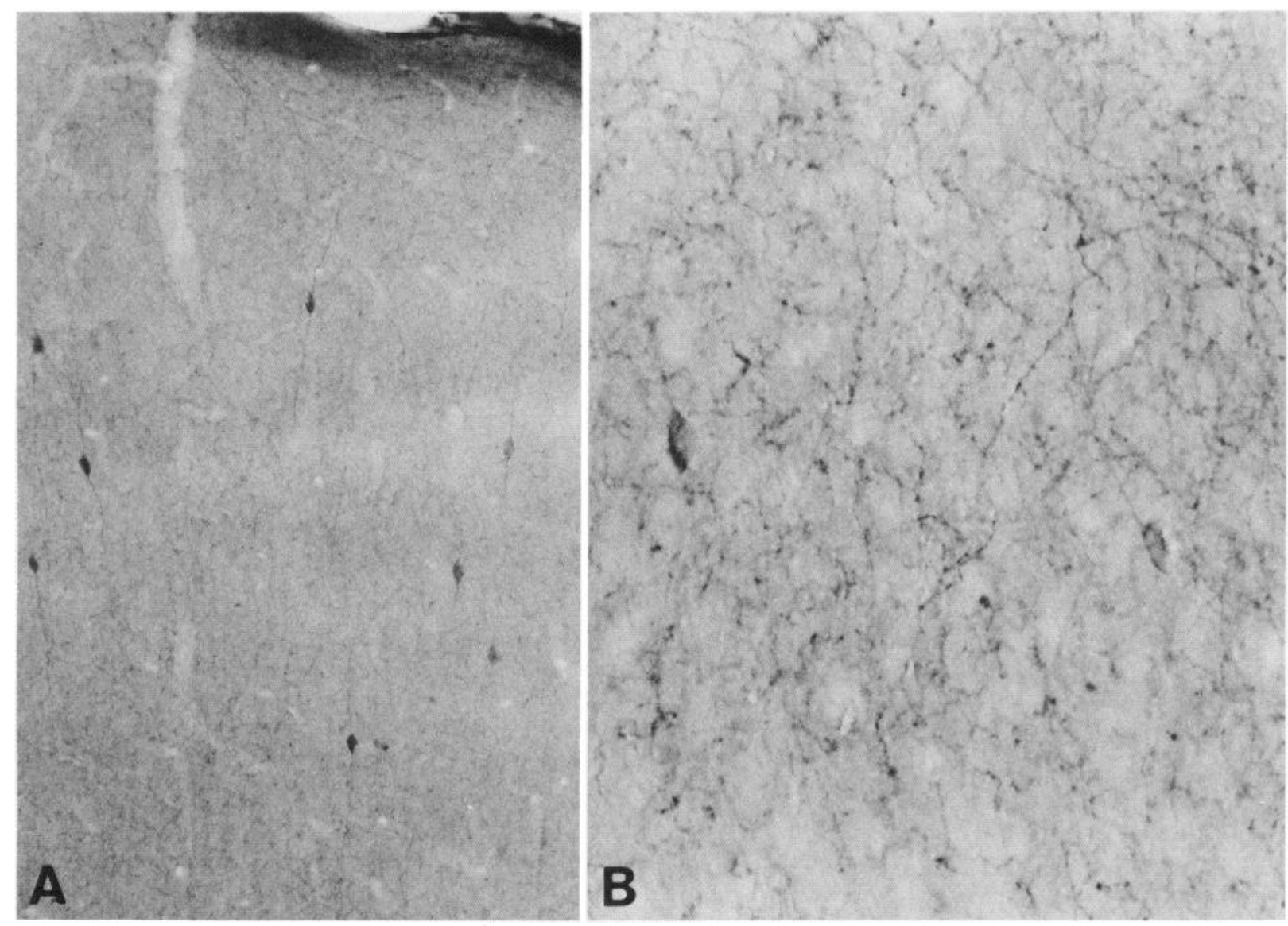

Figure 6. ChAT-positive structures in the neocortex. $A$, Neocortical ChAT-positivity at low magnification. ChAT-positive perikarya are seen in layers II-IV. Many of these exhibit a vertically oriented bipolar dendritic pattern $(\times 100)$. B, ChAT-positive varicose fiber network at higher magnification. Two neuronal perikarya are ChAT-positive $(\times 600)$.

peared to continue ventrally through the inferior thalamic peduncle to ChAT-positive perikaryal figures in the ventral part of the internal capsule (Fig. $9 B$ ) and the medial segment of the globus pallidus. The anteroventral nucleus presented finely granular ChAT-positivity but no fibrous figures were positive (Fig. $9, B, C)$.

\section{Hypothalamus}

A strongly ChAT-positive fiber bundle was observed in the ventromedial aspect of the suprachiasmatic nucleus (Fig. 9D). A few of these fibers were followed rostrally to the basal forebrain area. Within the nucleus, a ChAT-positive, fine fibrous meshwork was found, but it showed no preferential association with specific cellular elements. No distinct ChAT-positive structure was noticed in other areas of the hypothalamus. The ventromedial part of the hypothalamus, largely corresponding to the arcuate nucleus, showed an intense reactivity. The staining was, however, nonspecific: such staining was also observed in the control sections.

\section{Discussion}

A monoclonal antibody to rat ChAT, the specificity of which has been characterized (Ishida et al., 1983), was used in the present study to demonstrate the cholinergic structures in the forebrain of the rat immunohistochemically. The antibody revealed ChAT reactivity not only in perikarya, but also in proximal and distal dendrites, and axons and putative axon terminals, suggesting that the antibody stains essentially all major parts of the cholinergic neurons. Of the previous and present ChAT immunohistochemical studies, three staining patterns have been reported: (1) principally of perikarya and their proximal processes (Armstrong et al., 1983), (2) exclusively of axons and the presumed terminals (Chao et al., 1982), and (3) of cell bodies, dendrites, axons and probable terminals (Houser et al., 1983; Kimura et al., 1981a; the present study). The staining characteristic of the antibody used in the present observations provides detailed information, particularly on the trajectory of cholinergic fibers and their termination in the neuropil of the projection fields.

\section{Olfactory brain}

The present observation that there is no ChAT-positive perikaryon in the olfactory bulb is in agreement with that of Kimura et al. (1981a) in the cat. As for the cholinergic projection to the olfactory bulb, Kimura et al. (1981b) demonstrated the disappearance of ChAT-positive neurons in the ipsilateral nucleus of the diagonal band after lesioning of the olfactory bulb by electrocoagulation in the rat. Mesulam et al. (1983b) also indicated that the olfactory bulb is innervated by ChAT-positive neurons in the lateral part of the horizontal limb of the nucleus of the diagonal band in the rat. We provided additional evidence of this projection by demonstrating a ChAT-positive fiber bundle (rostral system) arising from the basal forebrain and entering the olfactory bulb both through the medial olfactory tract and the fibrillar layer of the anterior olfactory nucleus. At the termination sites of cholinergic afferents within the olfactory bulb, ChAT-positive varicose fibers were observed in the present study in every layer except for the olfactory nerve and medullary layers. This observation is generally consistent with the previous ChAT immunohistochemical studies in the cat by Kimura et 


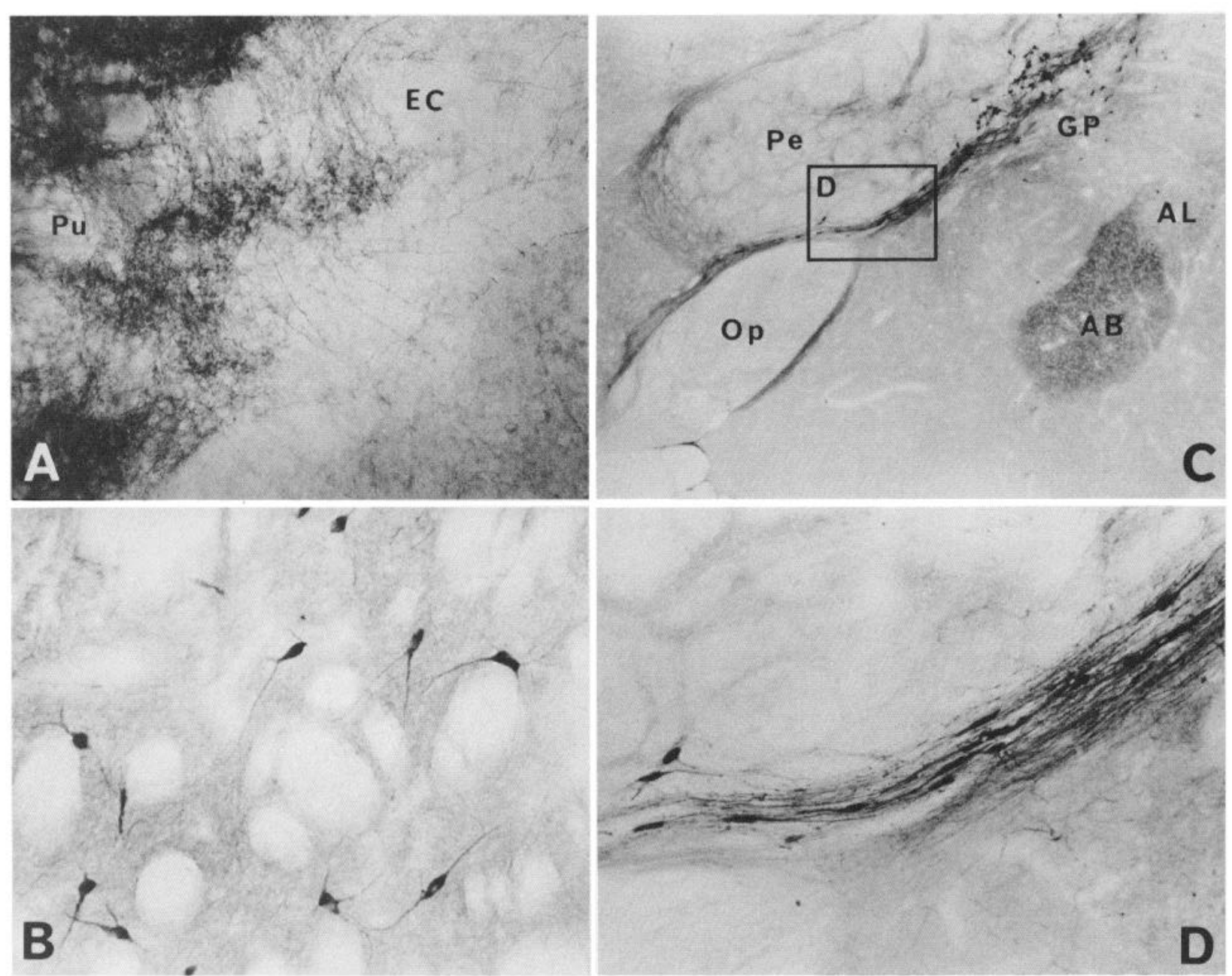

Figure 7. ChAT-positive structures in the caudate-putamen and globus pallidus. $A$, ChAT-positive fibers traversing the external capsule $(E C)$ are continuous to the granular positivity in the putamen $(P u)(\times 200)$. B, ChAT-positive perikarya in the caudate-putamen $(\times 150)$. $C$, ChAT-positive fibers from the globus pallidus $(G P)$ surround the cerebral peduncle $(P e)$ and run toward the ventrolateral nucleus. $A B$, lateral part of the basal nucleus of the amygdala; $A L$, posterior part of the lateral nucleus of the amygdala; $O p$, optic tract. Boxed area is reproduced at higher magnification in $D(\times 24)$. $D$, Elongated ChAT-positive perikarya are present among the fiber bundle surrounding the ventral surface of the cerebral peduncle $(\times 120)$.

al. (1981a) and the rabbit by Chao et al. (1982). A minor difference is that we could not find ChAT-positive structures in the medullary layer as described by Kimura et al. (1981a).

Unlike the observations of Kimura et al. (1981a) and Chao et al. (1982), we could not find ChAT-positive structures in the lateral olfactory tract, but we found them in the fibrillar layer of the anterior olfactory nucleus. Regarding the nucleus of the lateral olfactory tract, previous biochemical studies indicated high ChAT activity (Ben-Ari et al., 1977; Hoover et al., 1978; Palkovits et al., 1974), and we provided morphological evidence for this. The presence of interwoven ChAT-positive varicose fibers and the pericellular distribution of the varicosities strongly suggest that the nucleus is innervated by the cholinergic afferents, but their origin remains to be determined.

The distribution of ChAT-positive perikarya in the olfactory tubercle demonstrated in the present study is in agreement with that observed by others in the rat (Armstrong et al., 1983; Kimura et al., 1981b; Satoh et al., 1983; Sofroniew et al., 1982). The presence of ChAT-positive terminal-like structures in the olfactory tubercle and pyriform cortex has been reported by Kimura et al. (1981a, 1983) and Chao et al. (1982). We observed diffusely scattered granular profiles with ChAT-positivity in the olfactory tubercle, but their structural details remain to be elu- cidated. In the pyriform cortex, we observed ChAT-reactivity in varicose fibers. The varicosities, which had a pericellular distribution, may correspond to the ChAT-positive dots attached to surfaces of neurons reported by Kimura et al. (1983). Our observation of ChAT-positive stellate cells in the pyriform cortex is in agreement with that of Eckenstein and Thoenen (1983).

\section{Basal forebrain}

Our observation on the morphology and topographical localization of ChAT-positive perikarya is similar to those in previous studies on the rat (Armstrong et al., 1983; Houser et al., 1983; Kimura et al., 1981b; Satoh et al., 1983: Sofroniew et al., 1982), and is compatible with those on the cat (Kimura et al., 1981a), primate (Hedreen et al., 1983; Mesulam et al., 1984), and human (Nagai et al., 1983). We found no ChAT-positive structure in the neuropil, although Kimura et al. (1981a) reported that ChAT-positive dots are attached to the surfaces of ChAT-positive and -negative perikarya in the cat.

Cholinergic projection from the basal forebrain to the neocortex (Mesulam et al., 1983a, b; Wahle et al., 1984; Wainer and Rye, 1984; Woolf et al., 1983) and hippocampus (Mesulam et al., 1983a, b) has been indicated. Recently, Saper (1984) 

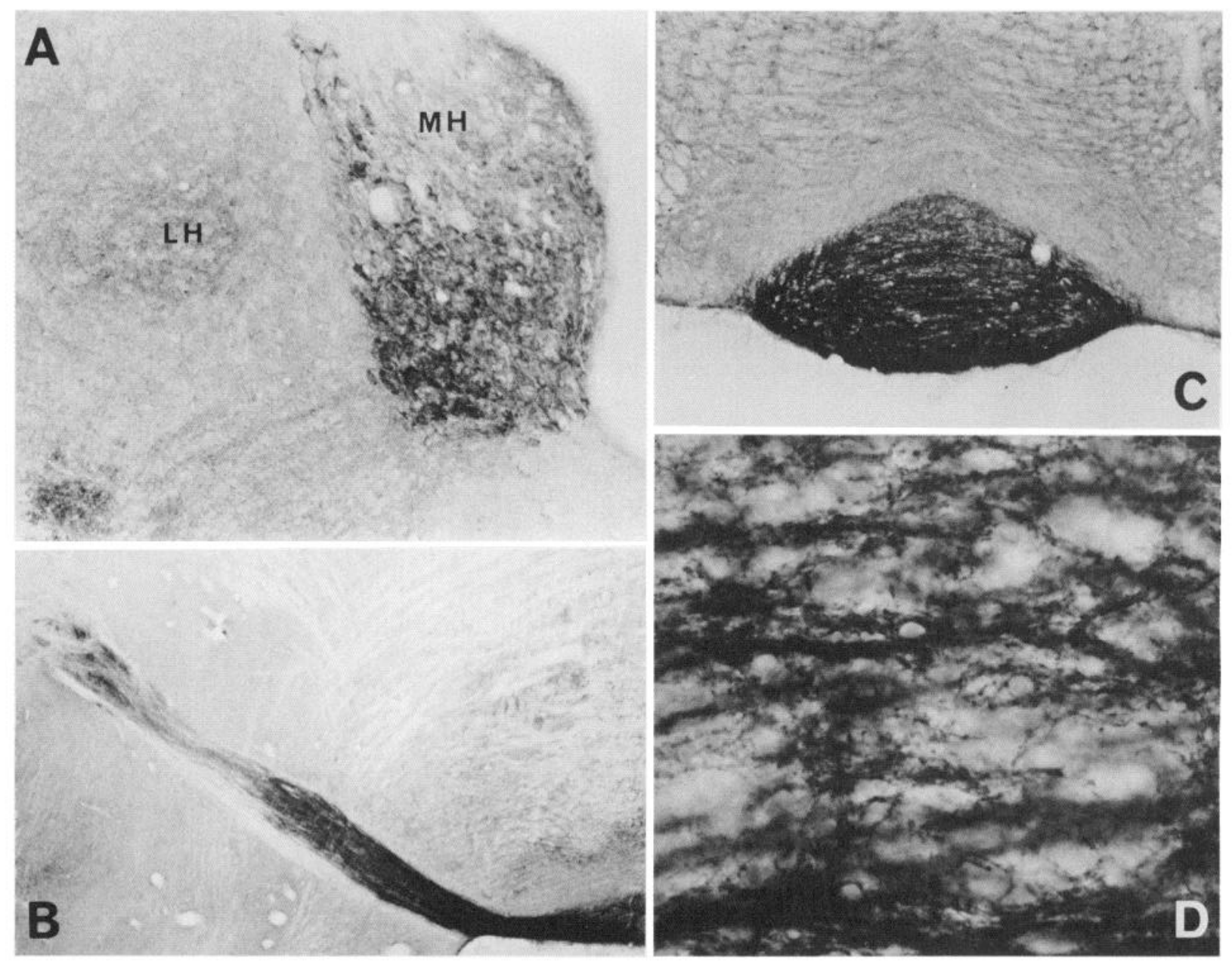

Figure 8. ChAT-positive structures in the habenulo-interpeduncular tract. $A$, ChAT-positive perikarya are seen in the medial habenular nucleus $(M H)$, while the lateral habenular nucleus $(L H)$ is free of staining $(\times 150)$. B, ChAT-positive fibers emerging from the ventrolateral aspect of the medial habenular nucleus descend in the fasciculus retroflexus (sagittal section, $\times 15$ ). $C$ and $D$, ChAT-positivity of the interpeduncular nucleus $(\times 30$ and $\times 400)$. A high-power micrograph $(D)$ shows ChAT-positive fiber bundles and ChAT-positive terminal-like dots budding from the fibers.

demonstrated two routes (medial and lateral pathways) from the basal forebrain to the neocortex and hippocampus by means of autoradiographic studies after injection of ${ }^{3} \mathrm{H}$-amino acids into the basal forebrain. He considered that these two pathways are cholinergic via cytoarchitectonic features obtained from ChAT-immunohistochemistry and AChE-histochemistry. In the present study, we have demonstrated the trajectory of the cholinergic fiber bundles from the basal forebrain to the neocortex and hippocampus. We found two systems, dorsomedial (to the neocortex and hippocampus) and dorsocaudal (to the hippocampus). The former corresponds well to the medial pathway described by Saper (1984). We could not, however, confirm his lateral pathway, and he did not describe our dorsocaudal system.

\section{Amygdaloid complex}

The cholinergic projection to the amygdaloid complex from the basal forebrain was indicated by Nagai et al. (1982) and Mesulam et al. (1983b). We observed ChAT-positive fibers entering the lateral nucleus and lateral large-celled part of the basal nucleus from the external capsule, but the route(s) from the basal forebrain to these nuclei was not demonstrated. These fibers were followed to ChAT-positive varicose fibers (lateral nucleus) and fine granular profiles (basal nucleus). Previous biochemical studies indicate that the lateral nucleus contains the highest ChAT activity, followed by the basal nucleus, with high activity, but that other nuclei are low in ChAT activity (Ben-Ari et al., 1977; Hoover et al., 1978; Palkovits et al., 1974). These results are compatible with our observations. As for the precise termination of the cholinergic afferents in the basolateral nucleus, Wainer et al. (1984) recently demonstrated boutons on the perikarya and dendritic shafts and spines via electron microscopic immunohistochemistry.

\section{Hippocampal formation}

Since we could not find any ChAT-positive perikarya in the hippocampal formation proper, most ChAT-positive fibers and terminals are thought to have an extrahippocampal origin. Houser et al. (1983) detected a few ChAT-positive neurons in this area, but they also described the cholinergic innervation to this region as predominantly from extrinsic neurons. Of ChATpositivity of Ammon's horn, that in the stratum oriens is thought to have come partly from the basal forebrain through the fornix (dorsomedial and dorsocaudal systems) since the positivity there often trails to fibrous positivity of the alveus-fimbria.

That the fibrous ChAT-positivity of the lacunosum-moleculare layers is followed to the transitional area from CA1 to the subiculum, and further to the subcortical white matter, suggests that this ChAT-positivity belongs to the perforant fiber system. Whether the pertinent perforant fibers are of cingulum origin or are from ChAT-positive neurons of the entorhinal-presubi- 
Figure 9. ChAT-positive structures in the dorsal thalamus and hypothalamus. $A, \mathrm{~A}$ low-power micrograph of a sagittal section. Boxed area is shown in $B(\times 8) . B$ and $C$, Fine granular ChAT-positivity in the reticular $(R T)$ and anteroventral $(A V)$ nuclei in sagittal $(B)$ and frontal sections $(C)(\times 24)$. ChAT-positive neuronal perikarya in the internal capsule $(I C)$ resemble those of the globus pallidus. $V A$, ventro-anterior nucleus. $D$, ChAT-positive fibers in the ventromedial aspect of the suprachiasmatic nucleus are continuous to ChAT-positive varicose fibers within the nucleus. $C h$, optic chiasm $(\times 80)$.
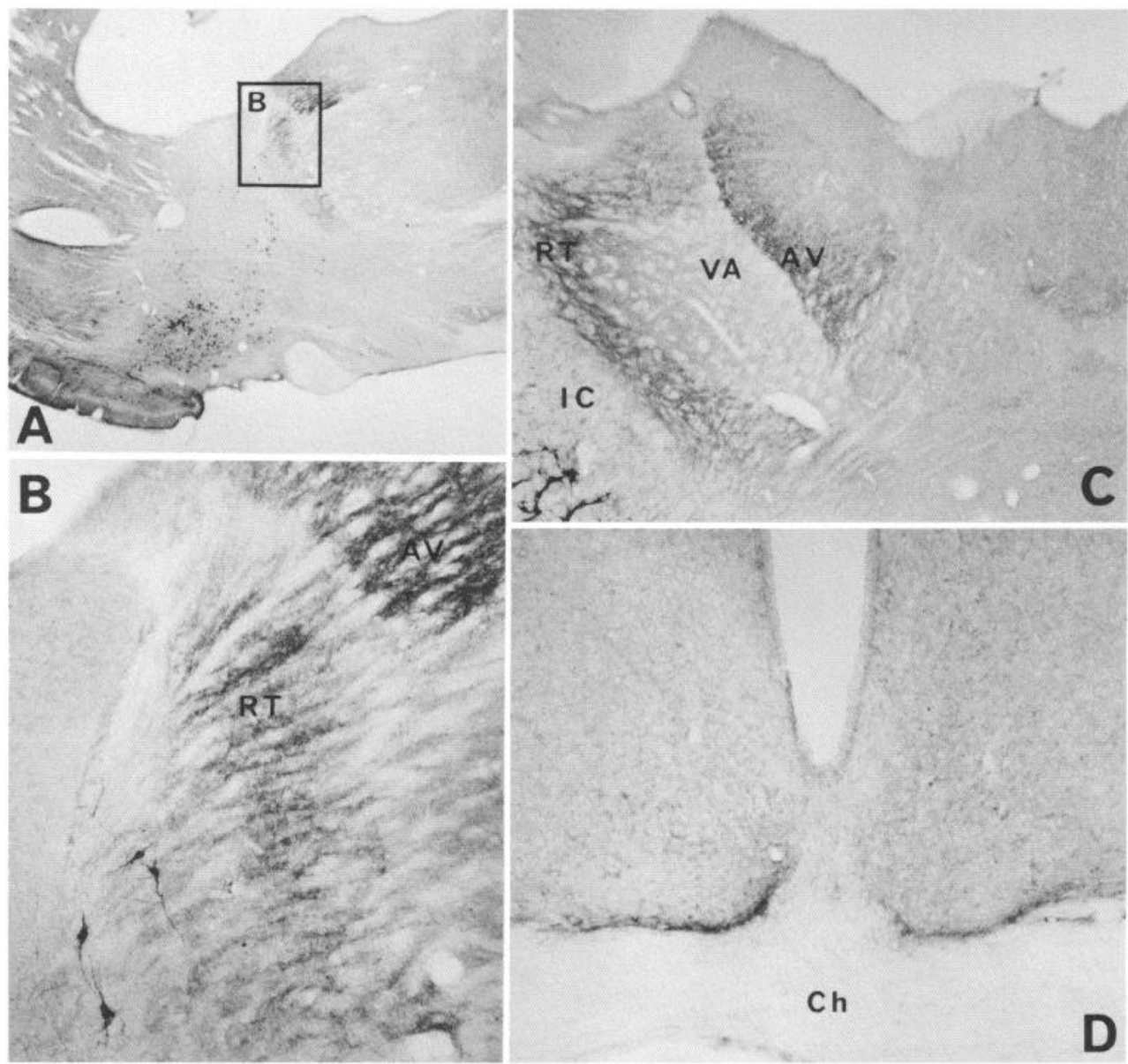

cular cortex, or from both, is difficult to determine from the present findings. But the presence of distinctly positive fibers in the cingulum favors the first possibility. Laminar distribution of ChAT-positivity in the molecular layer of the dentate fascia coincides with the ipsilateral entorhinal-dentate projection area (Cotman, 1978).

Peri- or juxta-perikaryal distribution of ChAT-reactivity in the fascia may represent the septal input, while the inner molecular layer corresponding to the projection area of the associational system (Cotman, 1978) is devoid of ChAT-reactivity.

\section{Neocortex}

ChAT-positive structures in the neuropil have been described by several investigators (Chao et al., 1982; Houser et al., 1983; Kimura et al., 1981a, 1983). Our results indicate that ChATpositive varicose fibers are scattered throughout all cortical layers and form a loose network. The observation is essentially the same as that of Houser et al. (1983). We could not observe the "terminal-like dots" described by Kimura et al. (1981a, 1983) either in the neuropil or on somatic surfaces. Chao et al. (1982) observed that ChAT-positive fibers and terminal-like structures are present mostly in layers V and VI, but such a preferential distribution was not confirmed in the present study. The diffuse distribution of cortical afferents observed in the present study suggests that this system exerts diverse spheres of influence on cortical elements. According to Wainer et al. (1984), cholinergic boutons contact dendritic shafts and spines in the cingulate cortex.

While several investigators observed ChAT-positive neurons in the cortex (Eckenstein and Baughman, 1984; Eckenstein and Thoenen, 1983; Houser et al., 1983), others failed to demonstrate neurons with ChAT-positivity (Armstrong et al., 1983;
Kimura et al., 1981a, b, 1983; Mesulam et al., 1984; Satoh et al., 1983; Sofroniew et al., 1982). Our observation corroborates the presence of cholinergic neuronal perikarya in the cortex. There is a good agreement concerning the appearance and localization of ChAT-positive neurons among the affirmative reports. Eckenstein and his colleagues at first failed to show ChATpositive neurons by the PAP method (Sofroniew et al., 1982), but they later succeeded in staining neurons in the neo- and pyriform cortices by the ABC method (Eckenstein and Thoenen, 1983). According to them, the discrepancy is probably due to differences in staining sensitivity between the two methods. Although our antibody revealed cortical ChAT-positive perikarya with both methods, we have noted that the staining intensity of the neurons by the $\mathrm{ABC}$ method is much stronger than that by the PAP one. Reexamination of the cortex using the ABC method by the investigators who could not stain cortical neurons is required to settle this difference.

Recently, Eckenstein and Baughman (1984) demonstrated vasoactive intestinal polypeptide (VIP)-immunoreactivity in ChAT-positive neurons in the cortex, and suggested that these ChAT- and VIP-positive neurons may be involved in regulating cortical blood flow. But we could not observe any particular association of ChAT-positive fibers and varicosities with blood vessels.

\section{Caudate-putamen, nucleus accumbens and globus pallidus}

The size, shape, and topography of ChAT-positive perikarya in the nuclei of the present study are akin to those in previous ones on the rat (Armstrong et al., 1983; Houser et al., 1983; Kimura et al., 1981b; Satoh et al., 1983; Sofroniew et al., 1982). These previous and the present studies consistently indicate that only 
a relatively small percentage of the neurons in the caudateputamen are cholinergic. Morphological features of ChAT-positive neurons have been studied electron microscopically by Sugimoto et al. (1984), using our antibody, and by Bolam et al. (1984). According to them, large aspiny type (Bishop et al., 1982) neurons are cholinergic. We observed that the neuropil of the caudate-putamen shows an intense ChAT-positivity, but the structural detail of ChAT-positive profiles was not clarified. Recently Wainer et al. (1984) observed immunoreactive synaptic boutons on the non-immunoreactive somata and dendritic shafts and spines. The neuropil of the nucleus accumbens shows an appearance similar to the caudate-putamen. The observation of granular ChAT-positive profiles in both regions is in agreement with that of Kimura et al. (1981a) and Houser et al. (1983).

As far as we know, the striatal cholinergic neurons have not been demonstrated to project outside the caudate-putamen and no extrinsic cholinergic afferents are known to innervate the striatum. However, the present demonstration that ChAT-positive fibers traverse the external capsule and enter the putamen suggests the presence of cholinergic striatal afferents and/or efferents. Further studies are needed to clarify this matter.

\section{Habenulo-interpeduncular tract}

The results of the previous immunohistochemical studies on this system have been inconsistent. According to Kimura et al. (1981a), the medial habenular nucleus is free of ChAT-positive perikarya, but it contains dense terminal-like dots. They also observed that fibers in the fasciculus retroflexus are ChAT-positive and the interpeduncular nucleus possesses terminal-like dots. Further, Armstrong et al. (1983) detected no ChAT-positive perikaryon in the habenula. In contrast, Houser et al. (1983) observed ChAT-positive perikarya in the medial habenular nucleus in addition to labeled fibers in the fasciculus retroflexus and terminal-like structures in the interpeduncular nucleus. The results of the last authors are in good agreement with ours. Observation on this system in the frontal and sagittal sections gives us an impression that the vast majority, if not all, of ChATpositive fibers in the fasciculus retroflexus arise from the medial habenular nucleus. As for the termination of this system in the interpeduncular nucleus, an electron microscopic immunohistochemical study was first carried out by Hattori et al. (1977), but the specificity of the antiserum employed was subject to criticism (Rossier, 1981). Using a monoclonal antibody, Wainer et al. (1984) recently showed ChAT-positive synaptic boutons that contact the dendritic shafts and spines.

\section{Thalamus}

There have been, thus far, no reports on ChAT-positive structures in the dorsal thalamus. In the present study, the thalamic reticular nucleus has shown the most prominent ChAT-positivity. The positivity in this nucleus is of a preterminal and terminal nature, hence being considered to represent a cholinergic afferent to this nucleus. The afferents to the reticular nucleus are known to come from the other thalamic nuclei (Jones, 1975) and cerebral cortex (Carman et al., 1964). Since no dorsal thalamic nuclei contain ChAT-positive perikarya, the possibility of a thalamic origin of cholinergic afferent is ruled out. The other possibility, of the neocortical cholinergic afferent to this nucleus, is rather small too, for most of the ChAT-positive cortical neurons are of small spindle or stellate type, lying mostly outside of the VIth layer, where the corticothalamic neurons are known to be located. Though hitherto not reported, pallidal ChATpositive neurons may be one of the origins of ChAT-positive afferents to the reticular nucleus, since some fibrous positivity can be traced to large ChAT-positive perikarya, seemingly of the pallidal neurons found ectopically in the internal capsule.

The anteroventral nucleus receives, in addition to the mamillothalamic projections, considerable amounts of afferent fibers from the limbic cortex (Berger et al., 1980 Kaitz and Robertson, 1981), where this nucleus projects. As in the case of the nucleus reticularis, the possibility of the corticothalamic afferent being responsible for ChAT-positive profiles in this nucleus is rather small. The source of ChAT-positive profiles seen in some intralaminar nuclei is difficult to determine, but it is probable that this ChAT-positivity is the continuation of ChAT-positive fibcrs running along the ventral aspect of the cerebral peduncle. Hence, these fibers might represent a fiber system from ChATpositive pallidal perikarya to the intralaminar-midline thalamic nuclei.

Recently, Mesulam et al. (1983b) indicated that the thalamic anterior, reticular, and lateral nuclei receive cholinergic innervation from ChAT-positive cell groups in the upper brainstem (Ch5 and Ch6). But we could not find ChAT-positive fiber bundles connecting these two regions.

\section{Hypothalamus}

Among the previous immunohistochemical investigations, $\mathrm{Ki}$ mura et al. (1981a) observed neurons with ChAT-positive dots on their surfaces throughout the entire hypothalamus, and Mason et al. (1983) showed that the supraoptic nucleus is innervated by ChAT-positive neurons lying just adjacent to the edge of the nucleus. In the present study, only the suprachiasmatic nucleus was shown to be definitely innervated by cholinergic afferents, probably of basal forebrain origin. The presence of ChAT in the suprachiasmatic nucleus has been demonstrated biochemically by Brownstein et al. (1975), and, recently, $\mathrm{Mu}$ rakami et al. (1984) demonstrated that light affects acetylcholine concentration in the nucleus. Although our observation suggests that the basal forebrain may be responsible for the innervation of the nucleus, the presence of the cholinergic retino-hypothalamic tract is not ruled out.

\section{References}

Armstrong, D. M., C. B. Saper, A. I. Levey, B. H. Wainer, and R. D. Terry (1983) Distribution of cholinergic ncurons in rat brain: demonstrated by the immunocytochemical localization of choline acetyltransferase. J. Comp. Neurol. 216: 53-68.

Ben-Ari, Y., R. E. Zigmond, C. C. D. Shute, and P. R. Lewis (1977) Regional distribution of choline acetyltransferase and acetylcholinesterase within the amygdaloid complex and stria terminalis system. Brain Res. 120: 435-445.

Berger, T. W., T. A. Milner, G. W. Swanson, G. S. Lynch, and R. F. Thompson (1980) Reciprocal anatomical connections between anterior thalamus and cingulate-retrosplenial cortex in the rabbit. Brain Res. 201: 411-417.

Bishop, G. A., H. T. Chang, and S. T. Kitai (1982) Morphological and physiological properties of neostriatal neurons: An intracellular horseradish peroxidase study in the rat. Neuroscience 7: 179-191.

Bolam, J. P., B. H. Wainer, and A. D. Smith (1984) Characterization of cholinergic neurons in the rat neostriatum. A combination of choline acetyltransferase immunocytochemistry, Golgi-impregnation and electron microscopy. Neuroscience 12: 711-718.

Brownstein, M., R. Kobayashi, M. Palkovits, and J. M. Saavedra (1975) Choline acetyltransferase levels in diencephalic nuclei of the rat. J. Neurochem. 24: 35-38.

Carman, J. B., W. M. Cowan, and T. P. S. Powell (1964) Cortical connexions of the thalamic reticular nucleus. J. Anat. 98: 587-598.

Chao, L.-P., K.-S. K. Kan, and F.-M. Hung (1982) Immunohistochemical localization of choline acetyltransferase in rabbit forebrain. Brain Res. 235: 65-82.

Cotman, C. W. (1978) Synapse formation and plasticity in the developing dentate gyrus. In Limbic Mechanisms, K. E. Livingston and O. Hornykiewicz, eds., pp. 47-65, Plenum, Ncw York and London.

Eckenstein, F., and R. W. Baughman (1984) Two types of cholinergic innervation in cortex, one co-localized with vasoactive intestinal polypeptide. Nature 309: 153-155.

Eckenstein, F., and M. V. Sofroniew (1983) Identification of central cholinergic neurons containing both choline acetyltransferase and ace- 
tylcholinesterase and of central neurons containing only acetylcholinesterase. J. Neurosci. 3: 2286-2291.

Eckenstein, F., and H. Thoenen (1983) Cholinergic neurons in the rat cerebral cortex demonstrated by immunohistochemical localization of choline acetyltransferase. Neurosci. Lett. 36: 211-215.

Fonnum, F. (1975) Review of recent progress in the synthesis, storage and release of acetylcholine. In Cholinergic Mechanisms, P. G. Waser, ed., pp. 145-160, Raven, New York.

Hattori, T., E. G. McGeer, V. K. Singh, and P. L. McGeer (1977) Cholinergic synapse of the interpeduncular nucleus. Exp. Neurol. 55: 666-679.

Hedreen, J. C., S. J. Bacon, L. C. Cork, C. A. Kitt, G. D. Crawford, P. M. Salvaterra, and D. L. Price (1983) Immunocytochemical identification of cholinergic neurons in the monkey central nervous system using monoclonal antibodies against choline acetyltransferase. Neurosci. Lett. 43: 173-177.

Hoover, D. B., E. A. Muth, and D. M. Jacobowitz (1978) A mapping of the distribution of acetylcholine, choline acetyltransferase and acetylcholinesterase in discrete areas of rat brain. Brain Res. 153: 295306.

Houser, C. R., G. D. Crawford, R. P. Barber, P. M. Salvaterra, and J. E. Vaughn (1983) Organization and morphological characteristics of cholinergic neurons: An immunocytochemical study with a monoclonal antibody to choline acetyltransferase. Brain Res. 266: 97-119.

Hsu, S.-M., L. Raine, and H. Fanger (1981) Use of avidin-biotinperoxidase complex $(\mathrm{ABC})$ in immunoperoxidase techniques: A comparison between $\mathrm{ABC}$ and unlabeled antibody (PAP) procedures. J. Histochem. Cytochem. 29: 577-580.

Ichikawa, T., I. Ishida, and T. Deguchi (1983) Monoclonal antibodies to choline acetyltransferase of rat brain. FEBS Lett. 155: 306-310.

Ishida, I., T. Ichikawa, and T. Deguchi (1983) Immunochemical and immunohistochemical studies on the specificity of a monoclonal antibody to choline acetyltransferase of rat brain. Neurosci. Lett. 42: 267-271.

Jones, E. G. (1975) Some aspects of the organization of the thalamic reticular complex. J. Comp. Neurol. 162: 285-308.

Kaitz, S. S., and R. T. Robertson (1981) Thalamic connections with limbic cortex. II. Corticothalamic projections. J. Comp. Neurol. 195: $527-545$.

Kimura, H., P. L. McGeer, J. H. Peng, and E. G. McGeer (1981a) The central cholinergic system studied by choline acetyltransferase immunohistochemistry in the cat. J. Comp. Neurol. 200: 151-201.

Kimura, H., P. L. McGeer, J. H. Peng, and E. G. McGeer (1981b) Mapping of cholinergic systems in rostral forebrain of the rodent. In Advances in Behavioral Biology. Vol. 25: Cholinergic Mechanisms: Phylogenetic Aspects, Central and Peripheral Synapses, and Clinical Significance, G. Pepeu and H. Ladinsky, eds., pp. 695-704. Plenum, New York and London.

Kimura, H., E. G. McGeer, F. Peng, and P. L. McGeer (1983) Cholinergic systems in the cat cortex studied by choline acetyltransferase immunohistochemistry. In Structure and Function of Peptidergic and Aminergic Neurons, Y. Sano, Y. Ibata and E. A. Zimmerman, eds., pp. 263-274, Japan Scientific Societies Press, Tokyo, and VNU Science Press, Utrecht.

Kuhar, M. J. (1976) The anatomy of cholinergic neurons. In Biology of Cholinergic Function, A. M. Goldberg and I. Hanin, eds., pp. 327, Raven, New York.

Levey, A. I., B. H. Wainer, E. J. Mufson, and M.-M. Mesulam (1983) Co-localization of acetylcholinesterase and choline acetyltransferase in the rat cerebrum. Neuroscience 9: 9-22.

Mason, W. T., Y. W. Ho, F. Eckenstein, and G. I. Hatton (1983) Mapping of cholinergic neurons associated with rat supraoptic nucleus: Combined immunocytochemical and histochemical identification. Brain Res. Bull. 11: 617-626.

McCaman, R. E., and M. W. McCaman (1976) Biology of individual cholinergic neurons in the vertebrate CNS. In Biology of Cholinergic Function, A. M. Goldberg and I. Hanin, eds., pp. 485-513, Raven, New York.

Mesulam, M.-M., E. J. Mufson, A. I. Levey, and B. H. Wainer (1983a) Cholinergic innervation of cortex by the basal forebrain: Cytochemistry and cortical connections of the septal area, diagonal band nuclci, nucleus basalis (substantia innominata), and hypothalamus in the rhesus monkey. J. Comp. Neurol. 214: 170-197.

Mesulam, M.-M., E. J. Mufson, B. H. Wainer, and A. I. Levey (1983b) Central cholinergic pathways in the rat: An overview based on an alternative nomenclature (Ch1-Ch6). Neuroscience 10: 1185-1201.

Mesulam, M.-M., E. J. Mufson, A. I. Levey, and B. H. Wainer (1984) Atlas of cholinergic neurons in the forebrain and upper brainstem of the macaque based on monoclonal choline acetyltransferase immunohistochemistry and acetylcholinesterase histochemistry. Neuroscience 12: 669-686.

Murakami, N., K. Takahashi, and K. Kawashima (1984) Effect of light on the acetylcholine concentrations of the suprachiasmatic nucleus in the rat. Brain Res. 311: 358-360.

Nagai, T., H. Kimura, T. Maeda, P. L. McGecr, F. Peng, and E. G. McGeer (1982) Cholinergic projections from the basal forebrain of rat to the amygdala. J. Neurosci. 2: 513-520.

Nagai, T., T. Pearson, F. Peng, E. G. McGeer, and P. L. McGeer (1983) Immunohistochemical staining of the human forebrain with monoclonal antibody to human choline acetyltransferase. Brain Res. 265 . 300-306.

Palkovits, M., J. M. Saavedra, R. M. Kobayashi, and M. Brownstein (1974) Choline acetyltransferase content of limbic nuclei of the rat. Brain Res. 79: 443-450.

Rossier, J. (1981) Serum monospecificity: A prerequisite for reliable immunohistochemical localization of neuronal markers including choline acetyltransferase. Neuroscience 6: 989-991.

Saper, C. B. (1984) Organization of cerebral cortical afferent systems in the rat. I. Magnoccllular basal nuclcus. J. Comp. Ncurol. 222: 313342.

Satoh, K., D. M. Armstrong, and H. C. Fibiger (1983) A comparison of the distribution of central cholinergic neurons as demonstrated by acetylcholinesterase pharmacohistochemistry and choline acetyltransferase immunohistochemistry. Brain Res. Bull. 11: 693-720.

Sofroniew, M. V., F. Eckenstein, H. Thoenen, and A. C. Cuello (1982) Topography of choline acetyltransferase-containing neurons in the forebrain of the rat. Neurosci. Lett. 33: 7-12.

Sugimoto, T., K. Mizukawa, T. Hattori, A. Konishi, T. Kaneko, and N. Mizuno (1984) Cholinergic neurons in the nucleus tegmenti pedunculopontinus pars compacta and the caudoputamen of the rat: A light and electron microscopic immunohistochemical study using a monoclonal antibody to choline acetyltransferase. Neurosci. Lett. 51: 113-117.

Wahle, P., C. Sanides-Buchholtz, F. Eckenstein, and K. Albus (1984) Concurrent visualization of choline acetyltransferase-like immunoreactivity and retrograde transport of neocortically injected markers in basal forebrain neurons of cat and rat. Neurosci. Lett. 44: 223228

Wainer, B. H., and D. B. Rye (1984) Retrograde horseradish peroxidase tracing combined with localization of choline acetyltransferase immunoreactivity. J. Histochem. Cytochem. 32: 439-443.

Wainer, B. H., J. P. Bolam, T. F. Freund, Z. Henderson, S. Totterdell, and A. D. Smith (1984) Cholinergic synapses in the rat brain: A correlated light and electron microscopic immunohistochemical study employing a monoclonal antibody against choline acetyltransferase. Brain Res. 308: 69-76.

Woolf, N. J., F. Eckenstein, and L. L. Butcher (1983) Cholinergic projections from the basal forebrain to the frontal cortex: A combined fluorescent tracer and immunohistochemical analysis in the rat. Neurosci. Lett. 40: 93-98. 